\title{
3 Von Griechen, Skythen und anderen
}

\begin{abstract}
Das Land, welches Pontus Euxinus genannt wird, [...] hat, das Skythenland ausgenommen, die unwissendsten Völker. Wir haben kein Volk innerhalb des Pontus, das wegen der Wissenschaft einigen Ruhm hätte [...]. Doch hat das skythische Volk eines der sonderbarsten Dinge, davon wir wissen, erfunden. Andere Dinge bewundere ich nicht. Das ist aber das sonderbarste [...], daß niemand, der sie überfällt, entfliehen, und daß sie auch niemand, wenn sie sich nicht finden lassen wollen, antreffen kann; denn die, die keine Städte und Mauern gebaut haben, die ihre Häuser mit sich herumführen, die Bogenschützen zu Pferd sind, die nicht von Ackerbau, sondern von der Viehzucht leben, die ihre Wohnungen auf Wagen haben, wie sollten diese nicht frei von allem Krieg und Überfall sein? ${ }^{1}$
\end{abstract}

Die bereits einleitend erwähnte Vorstellung, der nördliche Schwarzmeerraum und damit auch die Krim stellten eine Übergangszone zwischen Zivilisation und Barbarei dar, spiegelt sich in diesem Zitat Herodots aus dem fünften vorchristlichen Jahrhundert prägnant wider: Anders als die in diesem Absatz nicht extra erwähnten Griechen, zu denen sich der später als „Vater der Geschichtswissenschaft“ bezeichnete Herodot selbst zählte, hätten es die BewohnerInnen des Schwarzmeerraumes bzw. des Pontus Euxinus (griech. „das willkommen heißende Meer") nicht so mit der Gelehrsamkeit. Allein das nomadisch lebende skythische Volk habe es auf dem Gebiet der Kriegs- bzw. Kriegsvermeidungskunst zu einer offenbar erfolgreichen Taktik gebracht; dies hielt Herodot für umso lobenswerter, als sie damit die Gegner der Hellenen, die Perser, hatten schlagen können. Dass alle potentiellen Gegner ihrer nicht habhaft werden konnten, sie diese ihrerseits aber treffsicher aufspürten, nötigte dem Gelehrten eine gewisse Hochachtung ab. An anderer Stelle beschreibt er nüchtern, aber mit spürbarer Ablehnung über ihre für ihn barbarischen Sitten, dass jeder Krieger das Blut seines ersten getöteten Feindes trinke. Grundsätzlich brächten skythische Kämpfer die Köpfe getöteter Gegner dem König, da sich an deren Anzahl ihr Anteil an der Beute bemesse. Aus manchen abgeschlagenen Häuptern fertigten sie überdies mit Rinderleder überzogene Becher an, die zum Teil inwendig vergoldet würden. ${ }^{2}$

Die Auffassung, dass die am vermuteten Weltende siedelnden Fremden nicht nur anders, sondern auch grausam seien, ist in Herodots Beschreibung angedeutet. Allgemein gilt das sog. Fremde kultur- und epochenübergreifend nicht nur

1 Herodot, Historien, 4. Buch, 46, zitiert nach Herodot (o. J.), 295. Dieser ist keinesfalls der einzige Autor, in dessen Werk sich Hinweise zu den Skythen finden, zu erwähnen ist u. a. Hellanikos von Mytilene (490/480 v.Chr. - ca. 400 v.Chr.). Hinweise finden sich auch in den Tragödien von Sophokles oder Euripides.

2 Herodot, Historien, 4. Buch, 59-62, Schöll (1829), 469-473.

Ә OpenAccess. (c) 2020 Kerstin S. Jobst, publiziert von De Gruyter. (cc))BY Dieses Werk ist lizenziert unter der Creative Commons Attribution 4.0 International. https://doi.org/10.1515/9783110520620-005 
als brutal, sondern auch als wenig vernünftig, ja als irrational. ${ }^{3}$ So auch die Skythen, berichtete Herodot doch, diese seien abergläubisch, denn sie ließen sich „mit vielen Weidenruthen so wahrsagen.“4

Die neuere Herodot-Forschung will in dem griechischen Autor nicht mehr den geradezu prototypischen Vertreter der Auffassung sehen, dass das Eigene immer höherwertiger sei als das Fremde. ${ }^{5}$ Dennoch bleibt das Unbehagen spürbar, das der Autor empfunden haben muss, als er das ihm vertraute Leben der sesshaften Hellenen in der griechischen Polis mit dem der Skythen verglich, „die ihre Wohnungen auf Wagen haben."6 Andere Autoren, so z. B. der im 4. Jahrhundert v. Chr. lebende Ephoros von Kyme, werteten die Fremdheit und Exotik der Skythen demgegenüber eher positiv. ${ }^{7}$ Doch wen meinten die Griechen eigentlich, wenn sie über die „Skythen“ schrieben? Festzuhalten ist, dass die von Herodot und anderen Autoren dieser Zeit gebrauchte Bezeichnung „Skythen“ für die BewohnerInnen des nördlichen Schwarzmeerraums einschließlich der Krim nicht deren Eigenname war, sondern einfach eine allgemeine griechische Bezeichnung für alle Barbaren - d.h. Nicht-Griechen - in dieser zwischen Steppe und Küste gelegenen Region.

Der durch antike Autoren wie Herodot überlieferte Skythen-Begriff ist „nur chronologisch und kulturell zu definieren [...], nicht aber ethnisch“, wie der Prähistoriker Hermann Parzinger unterstreicht. ${ }^{8}$ Dies gilt grundsätzlich für mobile Personenverbände der Vormoderne, die eben nicht als ,Volk‘ oder gar ,Nationalität‘ zu bezeichnen sind. ${ }^{9}$ Das betrifft auch die vorskythischen Ankömmlinge auf der Krim, von denen wir nicht nur von Herodot Kenntnis haben, sondern auch von

3 Die Kategorien ,das Eigene، und ,das Fremde‘, auch in ihrer wechselseitigen Bedingtheit, werden seit langer Zeit in den Kulturwissenschaften diskutiert. Vgl. einführend Craanen u. Gunsenheimer (2006).

4 Herodot, Historien, 4. Buch, 67, zitiert nach Schöll (1829), 473.

5 So z.B. die Autoren Asheri, Lloyd und Corcella, welche sich gegen diese u.a. von Hartog F. (1980) vertretene Auffassung wenden, vgl. Asheri, u.a. (2007), 560, Anm. 33. Vgl. zur Essentialisierung im griechischen Altertum auch Hall E. (1989). Zu Herodots ethnographischen Beschreibungen vgl. Bichler (2000).

6 Hier nicht weiter auszuführen ist, dass Herodot in seinen Historien insgesamt drei Arten von Skythen unterscheidet: neben den nomadischen die sog. Königsskythen und die sog. Pflügerskythen.

7 Ivantchik (2005), 33.

8 Parzinger (2004), 123. Ein aktueller Forschungsüberblick ist folgender Publikation zu entnehmen: Chochorowski (2004).

9 An der von Wenskus (1977) vorgebrachten Anregung, stattdessen von gentilen Einheiten zu sprechen, haben sich viele WissenschaftlerInnen in der Folge orientiert. 
anderen griechischen Autoren. Berichtet wurde etwa von den Kimmeriern ${ }^{10}$, die ungefähr seit dem 8. Jahrhundert v. Chr. quellenmäßig fassbar geworden sind und deren eigentliche Herkunft Parzinger - wie die der Skythen auch - östlich des Urals verortet hat. ${ }^{11}$ Beide eurasischen Reiterkriegergemeinschaften sind nach allgemeiner Forschungsmeinung miteinander verwandt, nicht aber identisch. ${ }^{12}$

Die Skythen haben nicht zuletzt dank ihrer - bereits von Herodot hinlänglich beschriebenen - Begräbnisriten eine reichhaltige materielle Kultur hinterlassen. ${ }^{13}$ In archäologischen Funden aus skythischen Gräbern, Kurgane genannt, die vom eurasischen Raum bis weit nach Europa zu finden sind, fand sich beispielsweise feinst gearbeiteter goldener Körper- und Gewandschmuck ${ }^{14}$, der den Skythen zugeordnet wird. Dies konterkariert die Auffassung, es habe sich bei ihnen um kulturlose Barbaren gehandelt. Schwieriger sind Aussagen über die bereits im Kontext mit der „mythischen Krim“ (Kapitel 2) erwähnten Taurer zu treffen, die ebenfalls als vorskythisch gelten und in der südlichen Krim sowie in der Bergregion gelebt haben sollen. Trotz ihnen zugeordneter bronzezeitlicher Keramiküberreste ${ }^{15}$ werden sie primär durch antike Autoren fassbar, nicht aber durch andere Quellen. Herodot beispielsweise sah in ihnen das einzige auf der Krim lebende Volk, welches autochthon gewesen sei. ${ }^{16}$ Sie seien, wie für BewohnerInnen der Peripherie üblich, gefährlich, denn „[s]ie leben aber von Plünderung und Krieg. “17

Auf diese als vergleichsweise rückständig und gewaltbereit bezeichneten Barbaren - seien es nun Kimmerier, Taurer oder Skythen und später auch die bereits genannten Sarmaten - trafen im Schwarzmeerraum nun Hellenen. Diese

$10 \mathrm{Zu}$ den Kimmeriern vgl. die Arbeit von Sauter (2000), die schriftliche Berichte und archäologische Funde auswertet.

11 Parzinger (2004), 24.

12 Parzinger (2004), 18. Auch Ivantchik (2001) unterscheidet „Kimmerier“ territorial und literarisch voneinander.

13 Vgl. dazu Gossel-Raeck u. Busch (1993); Rolle (1991).

14 Parzinger (2004), 111f., spricht allerdings von einer wegen des Wirkens kenntnisreicher Grabräuber „gestörten Befundsituation.“

15 Chrapunow (1999), 21f. Dabei handelt es sich vor allen Dingen um Überreste, die nach dem südöstlich von Simferopol' gelegenen Fundort Kisil-Koba-Kultur benannt werden und aus dem 7.-

5. Jahrhundert v.Chr. stammen. Auch an der Südküste der Krim und in Bağçasaray wurde Keramik entdeckt, die auf eine taurische Besiedlung hinweist.

16 Dazu genauer Chrapunow (1999), 22. Diese Auffassung wird von einigen AutorInnen eingeschränkt, vgl. z. B. Magocsi (2014), der sich zu den Taurern folgendermaßen äußert: „The Taurans were a tribal group of unknown origin, who, at least from the first millennium BCE, lived in the mountainous zone of the Crimea where they engaged in animal husbandry, some agriculture, and fishing along the coasts“ (11).

17 Herodot, Historien, 4. Buch, 103, zitiert nach Schöll (1829), 497. 


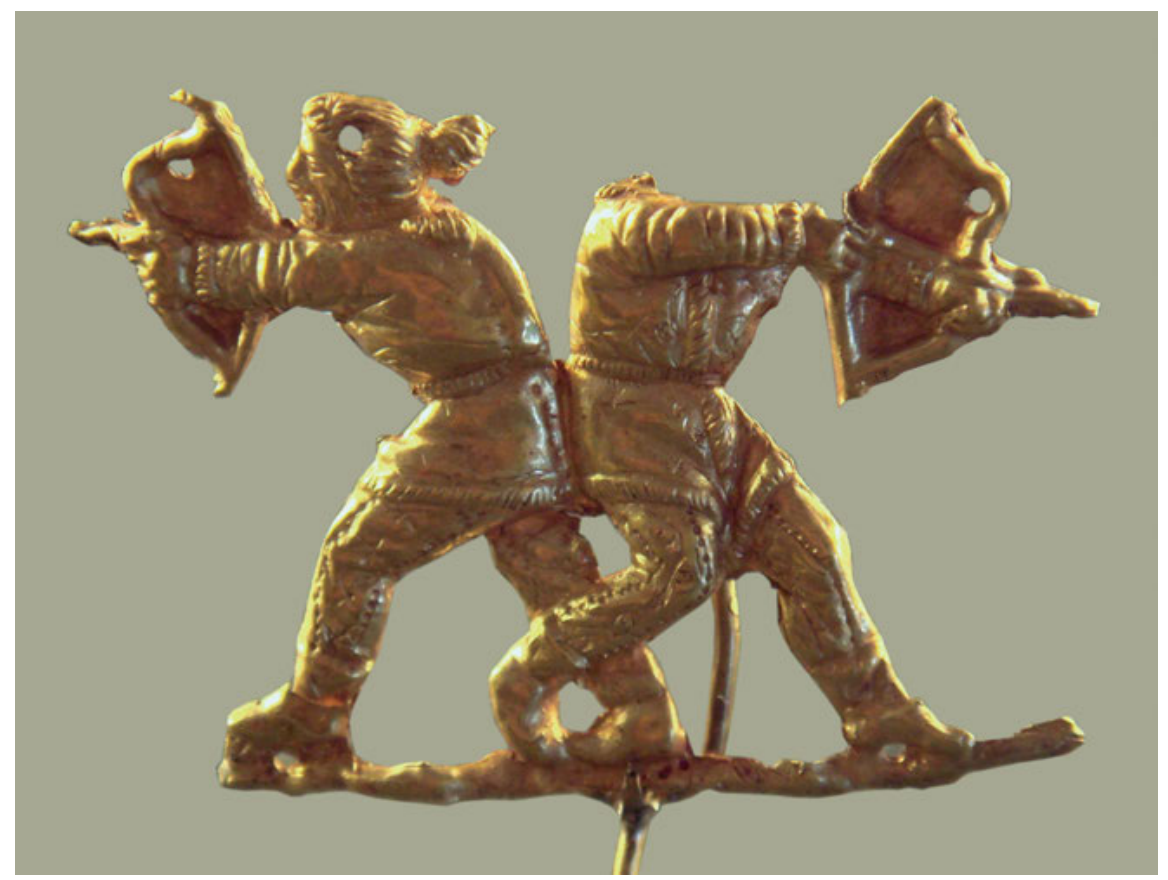

Abb. 3: Skythische Bogenschützen, Pantikapaion, 475-450 v. Chr.

traten mit diesen nicht nur in ökonomische, sondern in umfassende lebensweltliche Beziehungen, was dazu führte, dass die Barbaren und die Griechen sich über die Zeit partiell wechselseitig anverwandelten. Die Vorrausetzung dafür war das Einströmen einer größeren Zahl griechischer Kolonisten in das Gebiet. Für den Schwarzmeerraum und damit auch für die Krim war sowohl die sog. „Ionische“ (11./10. Jahrhundert v.Chr.) als auch die „Große Kolonisation“ (8. bis 6. Jahrhundert v.Chr.) von Bedeutung. In deren Folge entstanden auf der Halbinsel zahlreiche griechische Pflanzstädte (griech. Apoikien) - u.a. Chersones, das ursprünglich eine ionische Gründung und später eine dorische Neugründung war, bevor die heutige Ruinenstadt Teil eines Vororts von Sevastopol' geworden ist. Theodosia, das heutige Feodosija (russ./ukr.; krimtat. Kefe; auch Caffa oder Kaffa genannt), war eine Milet-Gründung, und Pantikapaion, vermutlich ebenfalls eine Milet-Gründung, ist in unserer Zeit das ganz im Osten der Halbinsel gelegene Kerč’ (krimtat. Keriç; ukr. Kerč).

Die griechischen Migranten im Schwarzmeerraum, die sog. Pontus-Griechen, waren ein wichtiges Element in der von damaligen Autoren geformten Erzählung 


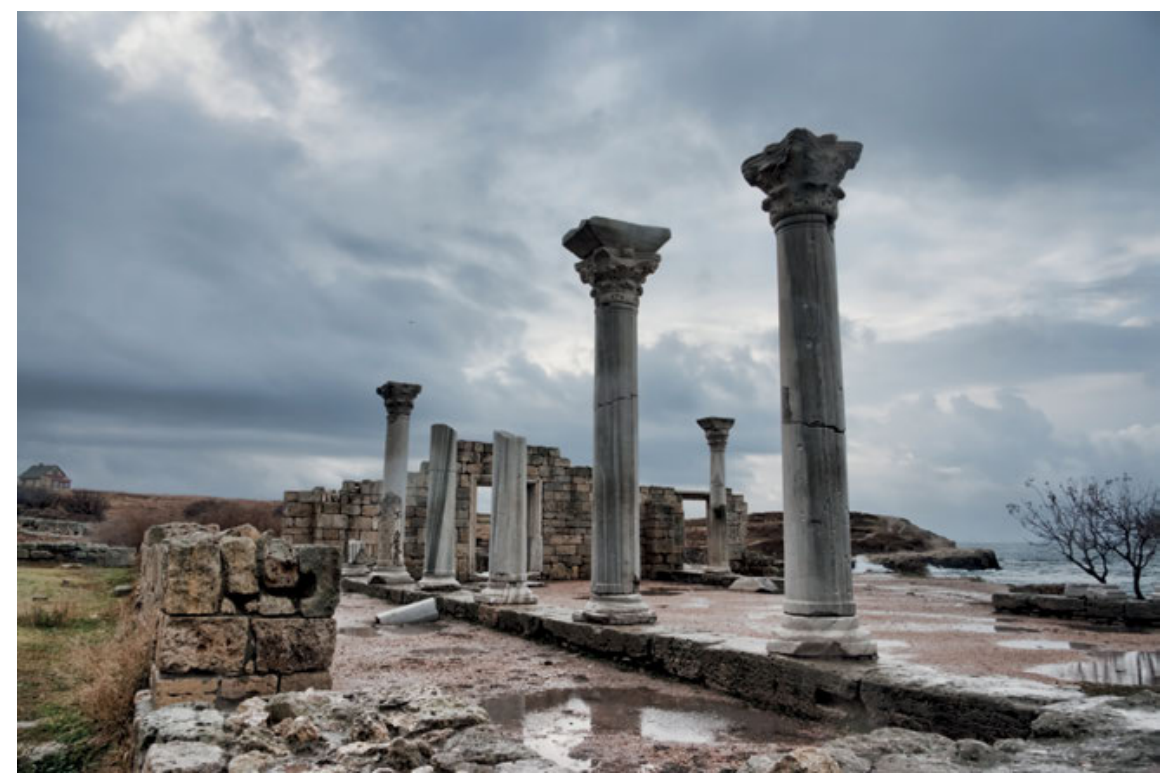

Abb. 4: Ruinen von Chersones

über die antike griechische kollektive Identität. ${ }^{18}$ Die Migration in die Gebiete außerhalb des hellenischen Kernlandes formten diese $\mathrm{zu}$ einem komplexen Siedlungsmythos, der den Glauben an die eigene Überlegenheit gegenüber der autochthonen Bevölkerung zu verfestigen half. Unabhängig davon, ob die jeweiligen Geschichten die Gründung einer griechischen Kolonie auf der iberischen Halbinsel, im Mittelmeer oder eben auf der Krim erzählten, weisen sie eine recht ähnliche Grundstruktur auf: Eine Mutterstadt entsendet eine beträchtliche Zahl von KolonistInnen in die Fremde, wo diese eine griechische Siedlung gründen, welche von der indigenen Bevölkerung hart bekämpft wird. Selbstverständlich obsiegen die überlegenen Hellenen am Ende über die Barbaren. ${ }^{19}$ Der realen Auswanderung aus dem Mutterland vorausgegangen waren - und dies ist der historische Hintergrund der griechischen Kolonistenbewegung - zumeist soziale und/oder politische Spannungen in der Metropole. In den legendisierten Erzählungen heißt es hingegen zumeist, Ausreisewillige hätten sich an das Orakel von Delphi gewandt, welches den Ratsuchenden die Auswanderung mit der Zustim-

18 Vgl. dazu Hall J. (2002), u. a. 45 f.

19 So das Ergebnis von Yntema (2010), 99. 
mung der Götter prophezeit habe. ${ }^{20}$ Diesem sehr linearen Erzählmuster stehen vielfach die archäologischen Befunde gegenüber, welche die Lesart einer ungebrochenen Erfolgsstory korrigieren. Über die sog. Große Kolonisation nach dem sechsten vorchristlichen Jahrhundert schreibt der Althistoriker Hermann Bengtson beispielsweise, bei dieser habe es sich um „eine unübersehbare Summe von vielfach unkontrollierbaren Einzelvorgängen, von Plänen, Versuchen, Erfolgen und Misserfolgen in bunter Reihe“ gehandelt. ${ }^{21}$ So erging es den Griechinnen und Griechen auch entlang der Küsten des Schwarzen Meeres einschließlich der Halbinsel Krim. Gleichwohl blieben sie, bis sie Opfer der stalinistischen Deportationen 1944 wurden, eine wichtige Akteursgruppe in der Region (vgl. dazu Kapitel 34). ${ }^{22}$

Wie hat man sich die Beziehungen der griechischen Kolonien entlang der nördlichen Schwarzmeerküste untereinander vorzustellen? Anfänglich waren sie miteinander nur wenig verbunden, pflegten hingegen intensive politische und ökonomische Kontakte zu ihren jeweiligen Mutterstädten; so blieben beispielsweise die im Osten der Halbinsel gelegenen ionischen Pflanzstädte wie Theodosia oder Pantikapaion mit Milet in engem Kontakt, während die ursprünglich aus Herakleia Pontike stammenden dorischen Kolonisten, die im Südwesten der Krim Siedlungen wie Chersones gegründet hatten, ihrerseits die Beziehungen zur Mutterstadt aufrechterhielten. In Zeiten externen und internen Drucks sowie auch aus wirtschaftlichen Erwägungen - insbesondere zur straffen Organisation des Getreidehandels - kam es parallel zu lokalen Zusammenschlüssen, zumindest wenn die einzelne Polis sich beispielsweise durch nomadischen Druck überfordert zeigte. Der wichtigste und lange Zeit auch ökonomisch florierende Städtebund wurde das um 480 v.Chr. entstandene und bis 362/375 n. Chr. existierende Bosporanische Königreich. ${ }^{23} \mathrm{Zu}$ beiden Seiten des sog. Kimmerischen Bosporus, der heute als Straße von Kerč' bezeichnet wird, formierte sich damit ein über die eigentliche Halbinsel hinausgehender Städtebund unter der Führung der Stadt Pantikapaion. Dieses Gemeinwesen orientierte sich nicht an der Tradition der attischen Demokratie, es war nämlich nicht republikanisch verfasst, sondern unterstand einem zwar offiziell gewählten Archonton (also einem führenden Amtsträger), welcher allerdings faktisch als Monarch fungierte und im vorlie-

20 Dazu Dougherty (1994).

21 Bengtson (1979), 67.

22 Nach der Rückeroberung der Krim durch die Rote Armee 1944 erlitten nämlich keineswegs nur die KrimtatarInnen das schwere Schicksal der Vertreibung, sondern auch andere über die Zeitläufte verwurzelte Gruppen wie die BulgarInnen oder eben auch die KrimgriechInnen.

23 Heinen (2006); Maslennikov (1981). Vgl. auch das ältere, aber noch immer wertvolle Werk Gajdukevič (1949). 
genden Fall eine erbliche Dynastie begründete. Nach den Archaianaktiden, einem vermutlich aus der Mutterstadt Milet stammenden Adelsgeschlecht, gelangte um das Jahr 438 v.Chr. die Familie der Spartokiden an die Macht, die sich bis zum Beginn des zweiten nachchristlichen Jahrhunderts halten konnte und die zwischenzeitlich eine durchaus expansive Politik verfolgte. ${ }^{24}$ Dass diese hellenischer Herkunft war, ist möglich, aber bislang nicht abschließend geklärt. Die Bevölkerung des Bosporanischen Königreiches war - wie auf der Krim und entlang der nördlichen Schwarzmeerküste insgesamt üblich - ethnisch und kulturell heterogen, trafen dort doch ,Neusiedler wie die griechischen KolonistInnen mit Gruppen zusammen, die dort schon länger siedelten. Der bedeutende sowjetische Archäologe Viktor F. Gajdukevič (1904-1966) bezeichnete das Bosporanische Reich deshalb und auf der Grundlage der Ergebnisse zahlreicher Ausgrabungen als „gräko-skythisch“25, was auf kulturelle und ethnische Synkretisierungsprozesse hinweist. Es ist davon auszugehen, dass zumindest die Eliten hellenischer Herkunft waren, wenngleich auch diese sich im andauernden Kulturkontakt mit skythischen (bzw. von griechischen Autoren als Skythen bezeichneten) Bevölkerungsschichten veränderten und - treffender vielleicht - als partiell hellenisch akkulturiert $\mathrm{zu}$ gelten haben. ${ }^{26}$

\section{Die Krim als polyethnischer Transit- und Siedlungsraum}

Ethnische, kulturelle und religiöse Vielgestaltigkeit, begleitet von ständigen Verschmelzungsprozessen, muss über die Zeitläufte als ein konstituierendes Kennzeichen des Schwarzmeerraumes und damit auch der Krim gelten. Erst das zwanzigste Jahrhundert markiert die tiefgreifende Zäsur in ihrer Geschichte, wurde doch die jahrtausendealte große ethnische und kulturelle Vielfalt beendet, die ein wesentliches Merkmal der ganzen Region dargestellt hatte - und dies seit frühesten Zeiten. ${ }^{27}$ Asiatische Gruppen (also Kimmerier, Skythen etc.) und griechische Kolonisten formten über die Zeit „mixed civilizations“, wie es der russische Altertumsforscher Michail I. Rostovcev (1870-1952) Anfang der 1920er Jahre ausgedrückt hat. ${ }^{28}$ Er verwarf damit die von griechischen Autoren angelegte und

24 Vgl. z. B. Selov-Kovedjaev (1986).

25 Gajdukevič (1949), 70.

$26 \mathrm{Zu}$ diesem Befund haben sowjetische Untersuchungen der Nekropolen erheblich beigetragen, vgl. Gajdukevič (1949), 233-297. Für die Ausgrabungen in den Jahren nach 1949 bis in die 1960er Jahre hinein vgl. die erweiterte deutschsprachige Ausgabe: Gajdukevič (1971).

27 Ich folge hier meinem Aufsatz Jobst (2015a).

28 Rostovtzeff (1922), 7. 
bei Herodot zumindest angedachte und über die Zeiten wirkmächtige Vorstellung der Unterscheidung zwischen zivilisierten, d.h. griechischen, und barbarischen Bevölkerungsgruppen. Damit stellte er die gerade in der ersten Hälfte des 20. Jahrhunderts populäre Vorstellung von der Existenz ,reiner' Kulturen in Frage, die allerdings in den russischen Geisteswissenschaften seit dem 19. Jahrhundert weitaus weniger stark ausgeprägt war als im europäischen Westen. Nicht wenige russische Intellektuelle im Zarenreich deuteten ihr ,asiatisches Erbe‘ nämlich durchaus positiv. ${ }^{29}$ So erklärt sich auch Rostovcevs Interesse, den von ihm zeittypisch als „Südrussland“ (und nicht etwa „Südukraine“) bezeichneten Raum zwischen Steppenregion und Schwarzmeerküste, zwischen Nomaden und Sesshaften, trotz seiner peripheren Lage als hochentwickelt und als Ergebnis komplexer kultureller Symbiosen zu beschreiben. Dies geschah auch in dem Bemühen, den Ostslaven ,ihren‘ Anteil an der von europäischen Intellektuellen seit der Aufklärung hochgeschätzten, ,wertvollen' Antike - und der Schwarzmeerraum war ja unbestritten Teil der antiken Welt - zu sichern. Auch spätere sowjetische Altertumsforscher betonten, dass „das Erbe der Völker des nördlichen Schwarzmeergebiets als ein Wesenselement in die Kultur der Ostslaven einging. So ist hier eine der Quellen der Kultur unserer Heimat.“30

Die Auffassung von der kulturellen Andersartigkeit bei paralleler Gleichwertigkeit nomadischer und sesshafter Kulturen wird von den modernen Kulturwissenschaften geteilt ${ }^{31}$; diese gehen heute ganz überwiegend davon aus, dass Nomaden und Sesshafte ,in der Geschichte ein Beziehungsgeflecht [bilden], in dem sich auf fast allen Ebenen gesellschaftlichen Handelns Differenzaspekte und Konfliktlinien mit integrativen Bewegungen von beiden Seiten überkreuzen. “32 Ob es sich dabei grundsätzlich, wie zuweilen formuliert worden ist, um ,asymmetrische Handelsbeziehungen“ gehandelt hat und damit ein „endemischer Konflikt“" zugrunde lag $^{33}$, soll hier nicht abschließend beurteilt werden. In den 1920er Jahren und den damals dominierenden nationalen und/oder rassi(sti)schen Diskursen waren Auffassungen wie die Rostovcevs aber eher umstritten. Tatsache

29 Darunter u.a. Fedor Dostoevskij oder im 20. Jahrhundert die sog. Eurasier. Vgl. hierzu einleitend Vucinich (1972); Hauner (1990).

30 D’jakov u. Nikol'skij (1952), $401 \mathrm{f}$.

31 Diese These untermauerte er u. a. mit Ergebnissen archäologischer Ausgrabungen skythischer Grabhügel, vgl. Rostovtzeff (1922), 20-25. Der Schwarzmeerraum einschließlich der Krim bietet somit HistorikerInnen und KulturwissenschaftlerInnen hervorragende Untersuchungsgegenstände für historische Zivilisationsvergleiche oder die Verflechtungsgeschichte, vgl. dazu Kaelble (1999); Werner u. Zimmermann (2004).

32 Leder (2005), 22.

33 So z. B. Kürşat-Ahlers (1994). 
ist, dass auf der Krim wie im ganzen nördlichen Schwarzmeerraum unterschiedlich wirtschaftende Gruppen in Kontakt traten und in einem komplexen, wechselseitigen Abhängigkeitsverhältnis standen, das gleichermaßen von Phasen der Koexistenz und Auseinandersetzung geprägt war. Auch deshalb entstand lange Zeit kein dauerhaft und überregional dominierendes Zentrum. Die Krim war und blieb in ihrer zwischen Steppe und Küste gelegenen Lage, an den Schnittstellen zwischen Imperien und Nomaden eine Peripherie - allerdings eine stark nachgesuchte, wie die anscheinend unablässig einwandernden, durchziehenden und bleibenden Bevölkerungsgruppen nachdrücklich belegen.

Offenbar bedarf das bereits in der Einleitung zitierte Diktum Aschersons, die Krim habe zu allen Zeiten den Wunsch ausgelöst, sie besitzen zu wollen, einer Erklärung. Warum zog die Halbinsel ungeachtet ihrer relativen Randlage so viele Völkerschaften an? Waren die klimatischen Gegebenheiten dort so ideal und/oder besaß die Halbinsel einen besonderen strategischen und ökonomischen Wert? Aktuell geht die Forschung davon aus, dass die Krim erdgeschichtlich eher jung ${ }^{34}$ und aufgrund ihrer günstigen Lage zwischen dem Schwarzen Meer und der Steppenregion sowie den vor allen Dingen an der Küste mediterranen klimatischen Verhältnisse bereits in vorantiker Zeit besiedelt gewesen ist. ${ }^{35}$ Allerdings sind die naturräumlichen Gegebenheiten auf der Halbinsel nicht überall so günstig wie im Süden, besteht sie doch aus drei unterschiedlichen landschaftlichen und klimatischen Zonen: erstens aus der Steppenlandschaft im Norden. Daran schließt sich eine mittlere Zone an, die durch das Krimgebirge geprägt wird, dessen höchste Erhebung mit 1.545 m der Roman-Koš (ukr./russ.; krimtat. Roman Qoş) ist. Südlich des Krimgebirges ermöglichte das mediterrane Klima schon im Altertum den Anbau von Weinstöcken und anderen wichtigen landwirtschaftlichen Produkten. Der nördliche Steppenraum schließlich war insofern ambivalent, als er den Transit von Waren aus dem eurasischen Raum genauso ermöglichte wie das Vordringen nomadischer Gruppen, welche in gewaltsame und gewaltarme Interaktionen mit den BewohnerInnen der anderen Zonen traten. Diese drei Zonen wurden von vielen AutorInnen, die über ihre Krim-Reisen berichteten, immer wieder nicht nur in nüchternen geographischen Kategorien beschrieben: So von Ascherson, der in der Krim einen lebendigen Organismus sieht, dessen Küsten den „Verstand“ repräsentieren, das Gebirge den „Geist“ und die Steppe den „Körper“. ${ }^{36}$ Der 1932 geborene schottische Autor bedient sich damit einer seit der Antike verbreiteten Metaphorik, durch die geographische Entitäten als Leib be-

34 Kushko (2014), 9.

35 Vgl. z. B. Stepančuk (1999).

36 Ascherson (1996), 35. 
schrieben werden. ${ }^{37}$ Die „Zone des Geistes“, also das Krimgebirge, stellt zwar ein gewisses geographisches Hindernis und eine Wetter- und Klimascheide zwischen der nördlichen Steppe und der Küste dar, war aber offenbar keine unüberwindbare Barriere für ursprünglich aus dem euroasiatischen Raum eindringende Völkerschaften wie die Skythen, die genauso Teil der Ökonomie und Lebenswelten des Schwarzen Meeres in der Antike wurden wie die Pontus-Griechen.

Die Zeit bis zum vierten vorchristlichen Jahrhundert gilt als Phase der Prosperität ${ }^{38}$ : Skythische Stämme und die diesen häufig tributpflichtigen griechischen Städte bildeten ein engmaschiges Produktions- und Handelsnetz aus, welches skythischerseits militärisch abgesichert wurde; grundsätzlich war das Verhältnis als pragmatisch $\mathrm{zu}$ bezeichnen, wobei Konflikte, welche die Geschäftsbeziehungen störten, ebenfalls auftraten. Neben Getreide, welches von großer Bedeutung für die Versorgung Athens war, ${ }^{39}$ Pelzen, Wachs und Honig wurden SklavInnen zu einem der wichtigsten Exportgüter. Die Krim versorgte einen großen Teil der antiken Welt mit diesen Gütern. Ascherson konstatiert, dass „[d]urch die mit Getreide und Sklavenhandel erzielten Profite [...] sowohl die griechischen Kaufleute wie die skythischen Fürsten im Oberland sehr reich“ wurden..$^{40}$ Dies war also für die Geschäftspartner, wie man heute sagen würde, eine ,Win-win-Situation'. Während des 3./2. Jahrhunderts v.Chr. setzte allerdings ein ökonomischer Niedergang ein, der sich u.a. am signifikanten Einbruch der Getreideproduktion zeigte. Dies war nicht zuletzt das Resultat des zunehmenden Drucks nördlicher und östlicher SteppenbewohnerInnen auf die Küstengebiete. Einer der bislang wichtigsten Kunden für Getreide von der Krim, Athen, war nach den Kriegen mit Makedonien in eine ökonomische Krise geraten, und die Handelsströme hatten sich verändert; $u$. a. machte Ägypten den Getreideproduzenten im Schwarzen Meer Konkurrenz. Hinzu kam das Vordringen neuer nomadischer und halbnomadischer Gruppen. Die ohnehin vielschichtige Gemengelage auf der Halbinsel veränderte sich allmählich.

37 Vgl. dazu grundlegend Baeque (1993).

38 Rostovtzeff (1922), $64 \mathrm{f}$.

39 Vgl. hierzu Moreno (2007), besonders das Kapitel „Ex Ponto. The Athenian Grain Supply and Black Sea Archaeology“, 144-208.

40 Ascherson (1996), $39 \mathrm{f}$. 